\title{
A MODELING OF COMPRESSIBLE DROPLETS IN A FLUID*
}

\author{
LAURENT BOUDIN ${ }^{\dagger}$, LAURENT DESVILLETTES $^{\ddagger}$, AND RENAUD MOTTE $§$
}

\begin{abstract}
In this work, we are interested in a complex fluid-kinetic model that aims to take into account the compressibility of the droplets of the spray. The ambient fluid is described by Euler-like equations, in which the transfer of momentum and energy from the droplets is taken into account, while the spray is represented by a probability density function satisfying a Vlasov-like equation. Implicit terms crop up because of the compressibility of the droplets. After having derived the model, we prove that global conservations are satisfied. Then we present two numerical tests. The first one enables us to validate the numerical code, while the second one is performed in a physically realistic situation.
\end{abstract}

\section{Introduction}

Complex two-phase fluids can be modelled in many different ways. The model known as (fully) Eulerian gives a description of both phases by physical quantities only depending on the variables $t$ and $x$ (typically, for example, the density, flow velocity and energy of each phase [RAV]).

We are here interested in a mixed fluid-kinetic modeling (a.k.a.particle-gas or Eulerian-Lagrangian), where the particles (or droplets) are described by a probability density function (PDF) solution of a kinetic equation in a phase space (including at least the variables $t, x$ and the velocities $u_{p}$ of the particles), whereas the continuous fluid satisfies traditional fluid equations. This type of model was introduced by Williams [WIA] (see also [CEC]). It is particularly suited to polydispersed flows, i.e. flows in which the size of the droplets can vary in a wide range. The same framework was used, for instance, by O'Rourke [ORO] (and his team in the Los Alamos National Laboratory), to develop the Kiva code [KIV], [KIW], and by many other authors (for example, Sainsaulieu [SAA], Domelevo [DOA] or Massot and Villedieu [MAS]).

This work is an attempt to take into account the compressibility of the droplets in a fluid-kinetic model at both modeling and numerical levels. This question has arisen in the framework of the study of a spray, in the context of the French military nuclear agency (CEA-DAM), after Motte's first approach [MOT]. The droplets remain spherical but their radii can vary. Note that, in some papers, e.g. [KIV], the assumption of sphericity is not systematically made. Moreover, we do not take into account the exchanges of mass between a particle and the ambient medium (vaporization, chemical reactions, etc.) or between two (or several) particles (collisions, coalescence, breakup $[\mathrm{BAA}] \ldots)$. That implies, in particular, that each particle has a constant mass. Moreover, at a thermodynamic level, we shall use the density $\rho$ and the specific internal energy $e$ as state variables, as in [MOT].

Note that we shall not tackle the problem of boundary conditions in this work.

In section 2 we derive the equations of our model and give some elements showing its coherence. Then, in section 3, we briefly discuss the numerical method and present

\footnotetext{
*Received: March 5, 2003; accepted (in revised version): September 8, 2003. Communicated by Peter Smereka.

${ }^{\dagger}$ Lab. J.-L. Lions - CNRS UMR 7598, Université Paris-VI, Boîte courrier 187, 75252 Paris CEDEX 05, FRANCE, (boudin@ann.jussieu.fr).

${ }^{\ddagger}$ CMLA - CNRS UMR 8536, ENS de Cachan, 61 avenue du Président Wilson, 94235 Cachan CEDEX, FRANCE, (desville@cmla.ens-cachan.fr).

§Centre d'études de Bruyères-le-Châtel, CEA/DAM, BP 12, 91680 Bruyères-le-Châtel, FRANCE, (renaud.motte@cea.fr).
} 
some numerical results.

\section{Presentation of the model}

2.1. The unknowns of the problem. We consider a complex two-phase flow made up of a fluid and droplets (or particles). The unknowns for the fluid are the volume fraction $\alpha(t, x)$, the density $\rho_{g}(t, x)$, the flow velocity $u_{g}(t, x)$, the mass internal energy $e_{g}(t, x)$, the temperature $T_{g}(t, x)$ and the fluid pressure $p(t, x)$.

The particles are described by the $\operatorname{PDF} f$. The value $f\left(t, x, u_{p}, e_{p}, m_{p}\right)$ is the number density of particles of mass $m_{p}$ located at coordinate $x$ at time $t$, moving with the velocity $u_{p}$ and having the internal energy $e_{p}$. Afterwards, since $m_{p}$ is a parameter which does not change, we shall write $f\left(t, x, u_{p}, e_{p}\right)$ instead of $f\left(t, x, u_{p}, e_{p}, m_{p}\right)$. We also assume the equality of pressures inside and outside a droplet, so that we do not introduce an extra quantity $p_{p}$. This assumption is classical (see [LAN] p. 57 or $[\mathrm{DRA}])$ : the pressures between two phases reaching an equilibrium is a very fast (mechanical) phenomenon with respect to the (thermodynamical) phenomenon of temperatures equilibrium. That implies in particular that the radius $r_{p}$ of a droplet and its density $\rho_{p}$ are not variables of the PDF, as in [KIV], but are functions of $e_{p}$, $m_{p}$ and the pressure $p(t, x)$. It is a significant difference compared to other already existing models (e.g. [KIV], [SAA]), in which one considers mass transfers, but not the compressibility of the particles, and where $r_{p}$ is a variable of the PDF.

2.2. Equation of state. The two equations of state for the fluid make it possible to obtain two algebraic relations between $p, \rho_{g}, e_{g}$ and $T_{g}$, that is to say

$$
\begin{aligned}
p(t, x) & =P_{1}\left(\rho_{g}(t, x), e_{g}(t, x)\right), \\
T_{g}(t, x) & =T_{1}\left(\rho_{g}(t, x), e_{g}(t, x)\right) .
\end{aligned}
$$

Moreover, one can define $\rho_{p}\left(t, x, e_{p}\right)$ by the formula

$$
p(t, x)=P_{2}\left(\rho_{p}\left(t, x, e_{p}\right), e_{p}\right)
$$

which is the pressure equation inside the droplet. Finally, one defines the temperature $T_{p}$ inside the droplet by

$$
T_{p}\left(t, x, e_{p}\right)=T_{2}\left(\rho_{p}\left(t, x, e_{p}\right), e_{p}\right) .
$$

That implies that $\rho_{p}$ does not depend on the variables $u_{p}$ and $m_{p}$. Moreover, since the mass of each droplet is invariant, $r_{p}$ is obviously a function of $t, x, e_{p}$ and $m_{p}$. More precisely, we have

$$
\frac{m_{p}}{\rho_{p}}=\frac{4}{3} \pi r_{p}^{3}
$$

2.3. The compressible model. The model considered here is an extension of the already existing model with incompressible droplets used by Motte in [MOT], in which we add the terms due to the compressibility of the particles.

2.3.1. Equations of the model. We propose the following system closed by the equations of state $(2.1)-(2.4)$ : 


$$
\begin{aligned}
& \partial_{t}\left(\alpha \rho_{g}\right)+\nabla_{x} \cdot\left(\alpha \rho_{g} u_{g}\right)=0 \\
& \partial_{t}\left(\alpha \rho_{g} u_{g}\right)+\nabla_{x} \cdot\left(\alpha \rho_{g} u_{g} \otimes u_{g}\right)+\nabla_{x} p=-\int_{u_{p}, e_{p}} m_{p} \Gamma f, \\
& \partial_{t}\left(\alpha \rho_{g} e_{g}\right)+\nabla_{x} \cdot\left(\alpha \rho_{g} e_{g} u_{g}\right)+p\left[\partial_{t} \alpha+\nabla_{x} \cdot\left(\alpha u_{g}\right)\right] \\
& =\int_{u_{p}, e_{p}}\left[\left(m_{p} \Gamma+\frac{m_{p}}{\rho_{p}} \nabla_{x} p\right) \cdot\left(u_{g}-u_{p}\right)-4 \pi r_{p} \lambda \mathrm{Nu}\left(T_{g}-T_{p}\right)\right] f, \\
& \alpha=1-\int_{u_{p}, e_{p}} \frac{m_{p}}{\rho_{p}} f, \\
& m_{p} \Gamma=-\frac{m_{p}}{\rho_{p}} \nabla_{x} p-D_{p}\left(u_{p}-u_{g}\right)+\rho_{g} C_{a} \frac{m_{p}}{\rho_{p}{ }^{2}} \frac{d \rho_{p}}{d t}\left(u_{p}-u_{g}\right) \text {, } \\
& m_{p} \Phi=4 \pi r_{p} \lambda \mathrm{Nu}\left(T_{g}-T_{p}\right)+\frac{p m_{p}}{\rho_{p}^{2}} \frac{d \rho_{p}}{d t}, \\
& \partial_{t} f+u_{p} \cdot \nabla_{x} f+\nabla_{u_{p}} \cdot(f \Gamma)+\partial_{e_{p}}(f \Phi)=0 \text {, }
\end{aligned}
$$

where

$$
\frac{d \rho_{p}}{d t}=\frac{\partial \rho_{p}}{\partial t}+u_{p} \cdot \nabla_{x} \rho_{p}+\Phi \frac{\partial \rho_{p}}{\partial e_{p}}
$$

Let us comment the choices carried out in (2.6)-(2.12).

Equations (2.6)-(2.8) come from the local conservation of mass, momentum and internal energy for the fluid. The right-hand sides model the feedback (transfer of momentum and energy) of the droplets on the fluid (we shall later detail their meaning while commenting equations (2.10)-(2.11)) and their form is usual [ORO], [KIV], [MOT]. One can recognize in the left hand sides of the equations the usual terms of the two-phase flow equations. Note that (2.7) can be rewritten, thanks to (2.9)-(2.10), under the more usual form

$\partial_{t}\left(\alpha \rho_{g} u_{g}\right)+\nabla_{x} \cdot\left(\alpha \rho_{g} u_{g} \otimes u_{g}\right)+\alpha \nabla_{x} p=\int_{u_{p}, e_{p}}\left[D_{p}\left(u_{p}-u_{g}\right)-\rho_{g} C_{a} \frac{m_{p}}{\rho_{p}{ }^{2}} \frac{d \rho_{p}}{d t}\left(u_{p}-u_{g}\right)\right] f$.

Equation (2.9) expresses the fact that the total volume is the sum of the volume of the fluid and the volume of the droplets. Moreover, thanks to this equation (2.9) and the fact that $f \geq 0$ (since $f$ is initially nonnegative and satisfies a Vlasov equation), it is clear that $\alpha \leq 1$. Although it is not obvious to mathematically prove that $\alpha \geq 0$, such models are assumed to be reliable only when $\alpha$ remains close to 1 (the volume of the droplets remains negligible to the volume occupied by the fluid). In the computations shown later, this is always verified.

Equations (2.6)-(2.9) together with (2.12) are more or less standard (cf. [ORO] for example).

The Vlasov equation (2.12) is obtained by writing down the conservation of the number of particles in a volume of the phase space (in $t, x, u_{p}$ and $e_{p}$ ). Note that one does not take into account the abrasion of the droplets and the mass transfers between both phases, whereas it is done in the Kiva code [KIV], for instance.

The form of relations (2.10) and (2.11) is specific to the case of compressible droplets. The quantities $\Gamma$ and $\Phi$ appear as the variations of $u_{p}$ and $e_{p}$ in the phase 
space, and are thus respectively given by the fundamental relation of dynamics (2.10) and by the equation of energy transfer (2.11) applied to a given particle.

In (2.10), the first two terms already appear when the droplets are incompressible: they represent the pressure force and the drag force. The last term is specific to the compressible case, and models an effect of mass addition. As a matter of fact, the volume variation of the droplet implies some local movements of the fluid which are very similar to a classical added mass force. The main term $\frac{m_{p}}{\rho_{p}{ }^{2}} \frac{d \rho_{p}}{d t}$ represents the volume variation of the droplets due to their compressibility and the remaining terms are obtained by analogy with the standard added mass force [CLG], [RAN]. Note that in the computations shown here, this term is very small with respect to the drag force.

In the equation of energy transfer (2.11), the term $4 \pi r_{p} \lambda \mathrm{Nu}\left(T_{g}-T_{p}\right)$ is already taken into account when the droplets are incompressible. It represents the heat transfer between both phases. The term with $d \rho_{p} / d t$ models the mechanical work due to the compressibility of the particles.

Eventually, the various physical coefficients $\left(\mathrm{Nu}, D_{p}\right.$, etc.) which appear here are defined in the appendix. They depend on the various quantities of the problem, such as $u_{p}, u_{g}$, etc.

Remark 1. The system (2.1)-(2.12) is implicit in the sense that there are time derivatives (of $\rho_{p}$ ) in right hand sides of some equations. Those can be written in terms of $\partial_{t} p$ because of (2.3), then in terms of $\partial_{t} \rho_{g}, \partial_{t} e_{g}$ because of (2.1), of $\partial_{t} \alpha$ because of (2.6)-(2.8), and finally of $\partial_{t} \rho_{p}$ itself because of (2.9) (and (2.10)-(2.11)). Thus one can prove that

$$
\partial_{t} \rho_{p}\left(t, x, \tilde{e_{p}}\right)=\int_{u_{p}, e_{p}} \mathcal{F}\left(t, x, u_{p}, e_{p}, \tilde{e_{p}}\right) \partial_{t} \rho_{p}\left(t, x, e_{p}\right) f\left(t, x, u_{p}, e_{p}\right) d u_{p} d e_{p}+\mathcal{G}\left(t, x, \tilde{e_{p}}\right),
$$

where there are no time derivatives in the expressions of $\mathcal{F}$ and $\mathcal{G}$.

We only detail the expression of $\mathcal{F}$ in the one-dimensional case (the expression of $\mathcal{G}$ is even more intricate than the one of $\mathcal{F}$, so we choose not to write it down). We introduce the state equations derivative coefficients

$$
\begin{gathered}
A_{g}(t, x)=\left(\frac{\partial p}{\partial \rho}\right)_{\text {fluid }}\left(\rho_{g}(t, x), e_{g}(t, x)\right), B_{g}(t, x)=\left(\frac{\partial p}{\partial e}\right)_{\text {fluid }}\left(\rho_{g}(t, x), e_{g}(t, x)\right), \\
A_{p}\left(t, x, e_{p}\right)=\left(\frac{\partial \rho}{\partial p}\right)_{\text {drop }}\left(p(t, x), e_{p}\right), B_{p}\left(t, x, e_{p}\right)=\left(\frac{\partial \rho}{\partial e}\right)_{\text {drop }}\left(p(t, x), e_{p}\right), \quad(2.14
\end{gathered}
$$

and the following coefficient (which equals $\gamma$ when the droplets constitute an ideal gas)

$$
\zeta_{p}\left(t, x, e_{p}\right)=\left(1-\frac{p(t, x)}{\rho_{p}\left(t, x, e_{p}\right)^{2}} B_{p}\left(t, x, e_{p}\right)\right)^{-1} .
$$

For the sake of simplicity, we do not write the dependence of the functions in the variables $t$ and $x$. We can then write down

$$
\mathcal{F}\left(u_{p}, e_{p}, \tilde{e_{p}}\right)=-\frac{A_{p}\left(\tilde{e_{p}}\right)}{\alpha} \frac{m_{p} \zeta_{p}\left(e_{p}\right)}{\rho_{p}\left(e_{p}\right)^{2}}\left[A_{g} \rho_{g}+B_{g}\left(\frac{p}{\rho_{g}}+C_{a}\left|u_{p}-u_{g}\right|^{2}\right)\right] .
$$

We shall explain in Section 3 how this difficulty is tackled at the numerical level. 
2.3.2. Global conservations. First note that the system (2.1)-(2.12) is closed. Like for most of the systems describing complex fluids, we verify that the properties of conservation hold.

Proposition 1. The total energy, momentum and mass of the system (2.1)-(2.12) are conserved.

Proof. For the convenience of the proof, we set

$$
\mathcal{H}_{p}=\frac{p m_{p}}{\rho_{p}{ }^{2}} \frac{d \rho_{p}}{d t}
$$

so that equation (2.11) writes

$$
m_{p} \Phi=4 \pi r_{p} \lambda \mathrm{Nu}\left(T_{g}-T_{p}\right)+\mathcal{H}_{p}
$$

We successively verify the conservations of the total energy, momentum and mass of the system. We first notice that

$$
\alpha \rho_{g} \partial_{t} u_{g}=-\alpha \rho_{g}\left(\nabla_{x} \cdot u_{g}\right) u_{g}-\nabla_{x} p-\int_{u_{p}, e_{p}} m_{p} \Gamma f
$$

by using (2.6) and (2.7) together. Moreover, by using (2.12) and (2.14), the derivation of (2.9) with respect to $t$ gives

$$
\begin{aligned}
\partial_{t} \alpha & =\int_{u_{p}, e_{p}} m_{p}\left(\frac{\partial_{t} \rho_{p}}{\rho_{p}{ }^{2}} f-\frac{1}{\rho_{p}} \partial_{t} f\right) \\
& =\int_{u_{p}, e_{p}} m_{p}\left[\frac{\partial_{t} \rho_{p}}{\rho_{p}{ }^{2}} f+\frac{1}{\rho_{p}}\left(u_{p} \cdot \nabla_{x} f+\nabla_{u_{p}} \cdot(f \Gamma)+\partial_{e_{p}}(f \Phi)\right)\right] \\
& =\int_{u_{p}, e_{p}} m_{p} \frac{\partial_{t} \rho_{p}}{\rho_{p}{ }^{2}} f+\int_{u_{p}, e_{p}} \frac{m_{p}}{\rho_{p}} u_{p} \cdot \nabla_{x} f+\int_{u_{p}, e_{p}} \frac{m_{p} \Phi}{\rho_{p}{ }^{2}} B_{p} f .
\end{aligned}
$$

Now let us compute the variations of the total energy $E$ of the system

$$
\begin{aligned}
\frac{d E}{d t}= & \frac{d}{d t} \int_{x} \alpha \rho_{g}\left(e_{g}+\frac{\left|u_{g}\right|^{2}}{2}\right)+\frac{d}{d t} \int_{x, u_{p}, e_{p}} m_{p}\left(e_{p}+\frac{\left|u_{p}\right|^{2}}{2}\right) f \\
= & \int_{x} \partial_{t}\left(\alpha \rho_{g} e_{g}\right)+\frac{1}{2} \int_{x} \partial_{t}\left(\alpha \rho_{g} u_{g}\right) \cdot u_{g}+\frac{1}{2} \int_{x} \alpha \rho_{g} u_{g} \cdot \partial_{t} u_{g} \\
& +\int_{x, u_{p}, e_{p}} m_{p}\left(\frac{\left|u_{p}\right|^{2}}{2}+e_{p}\right) \partial_{t} f .
\end{aligned}
$$

By using (2.8), (2.7), (2.16) and (2.12) and by directly eliminating the integrated 
conservative terms, one obtains

$$
\begin{aligned}
\frac{d E}{d t}= & -\int_{x} p \partial_{t} \alpha-\int_{x} p \nabla_{x} \cdot\left(\alpha u_{g}\right) \\
& +\int_{x, u_{p}, e_{p}} m_{p} \Gamma \cdot\left(u_{g}-u_{p}\right) f+\int_{x, u_{p}, e_{p}} \frac{m_{p}}{\rho_{p}} \nabla_{x} p \cdot\left(u_{g}-u_{p}\right) f \\
& -\int_{x, u_{p}, e_{p}} 4 \pi r_{p} \lambda \mathrm{Nu}\left(T_{g}-T_{p}\right) f \\
& +\frac{1}{2} \int_{x} u_{g} \cdot\left[-\nabla_{x} \cdot\left(\alpha \rho_{g} u_{g} \otimes u_{g}\right)-\nabla_{x} p-\int_{u_{p}, e_{p}} m_{p} \Gamma f\right] \\
& +\frac{1}{2} \int_{x} u_{g} \cdot\left(-\alpha \rho_{g}\left(\nabla_{x} \cdot u_{g}\right) u_{g}-\nabla_{x} p-\int_{u_{p}, e_{p}} m_{p} \Gamma f\right) \\
& -\int_{x, u_{p}, e_{p}} m_{p}\left(\frac{\left|u_{p}\right|^{2}}{2}+e_{p}\right)\left(\nabla_{u_{p}} \cdot(f \Gamma)+\partial_{e_{p}}(f \Phi)\right) .
\end{aligned}
$$

With (2.16)-(2.17), we find

$$
\begin{aligned}
\frac{d E}{d t}= & -\int_{x, u_{p}, e_{p}} p \frac{m_{p}}{\rho_{p}{ }^{2}} \partial_{t} \rho_{p} f-\int_{x, u_{p}, e_{p}} p \frac{m_{p}}{\rho_{p}} u_{p} \cdot \nabla_{x} f-\int_{x, u_{p}, e_{p}} p \frac{m_{p} \Phi}{\rho_{p}{ }^{2}}\left(\frac{\partial \rho_{p}}{\partial e_{p}}\right) f \\
& +\int_{x} \alpha u_{g} \cdot \nabla_{x} p+\int_{x, u_{p}, e_{p}} m_{p} \Gamma \cdot\left(u_{g}-u_{p}\right) f \\
& +\int_{x, u_{p}, e_{p}} \frac{m_{p}}{\rho_{p}} \nabla_{x} p \cdot\left(u_{g}-u_{p}\right) f-\int_{x, u_{p}, e_{p}} 4 \pi r_{p} \lambda \mathrm{Nu}\left(T_{g}-T_{p}\right) f \\
& -\int_{x} u_{g} \cdot \nabla_{x} p-\int_{x, u_{p}, e_{p}} m_{p} \Gamma \cdot u_{g} f+\int_{x, u_{p}, e_{p}} m_{p} \Gamma \cdot u_{p} f+\int_{x, u_{p}, e_{p}} m_{p} \Phi f .
\end{aligned}
$$

Thanks to an integration by parts in the second integral, and using (2.9) and (2.15), we get

$$
\begin{aligned}
\frac{d E}{d t}= & -\int_{x, u_{p}, e_{p}} p \frac{m_{p}}{\rho_{p}{ }^{2}} \partial_{t} \rho_{p} f+\int_{x, u_{p}, e_{p}} m_{p} u_{p} \cdot \nabla_{x}\left(\frac{p}{\rho_{p}}\right) f \\
& -\int_{x, u_{p}, e_{p}} \frac{p}{\rho_{p}^{2}} m_{p} \Phi\left(\frac{\partial \rho_{p}}{\partial e_{p}}\right) f-\int_{x, u_{p}, e_{p}} \frac{m_{p}}{\rho_{p}} \nabla_{x} p \cdot u_{g} f \\
& +\int_{x, u_{p}, e_{p}} \frac{m_{p}}{\rho_{p}} \nabla_{x} p \cdot\left(u_{g}-u_{p}\right) f+\int_{x, u_{p}, e_{p}} \mathcal{H}_{p} f
\end{aligned}
$$

hence

$$
\begin{aligned}
\frac{d E}{d t}= & -\int_{x, u_{p}, e_{p}} p \frac{m_{p}}{\rho_{p}{ }^{2}} \partial_{t} \rho_{p} f+\int_{x, u_{p}, e_{p}} \frac{m_{p}}{\rho_{p}} u_{p} \cdot \nabla_{x} p f \\
& -\int_{x, u_{p}, e_{p}} p \frac{m_{p}}{\rho_{p}^{2}} u_{p} \cdot \nabla_{x} \rho_{p} f-\int_{x, u_{p}, e_{p}} \frac{p}{\rho_{p}^{2}} m_{p} \Phi\left(\frac{\partial \rho_{p}}{\partial e_{p}}\right) f \\
& -\int_{x, u_{p}, e_{p}} \frac{m_{p}}{\rho_{p}} u_{p} \partial_{x} p f+\int_{x, u_{p}, e_{p}} \mathcal{H}_{p} f \\
= & 0 .
\end{aligned}
$$


The total mass conservation holds

$$
\begin{aligned}
& \frac{d}{d t} \int_{x} \alpha \rho_{g}+\frac{d}{d t} \int_{x, u_{p}, e_{p}} m_{p} f \\
& \quad=\int_{x} \partial_{t}\left(\alpha \rho_{g}\right)+\int_{x, u_{p}, e_{p}} m_{p} \partial_{t} f \\
& \quad=-\int_{x} \nabla_{x} \cdot\left(\alpha \rho_{g} u_{g}\right)-\int_{x, u_{p}, e_{p}} m_{p}\left(u_{p} \cdot \nabla_{x} f+\nabla_{u_{p}} \cdot(f \Gamma)+\partial_{e_{p}}(f \Phi)\right) \\
& \quad=0
\end{aligned}
$$

and so does the total momentum conservation

$$
\begin{aligned}
& \frac{d}{d t} \int_{x} \alpha \rho_{g} u_{g}+\frac{d}{d t} \int_{x, u_{p}, e_{p}} m_{p} u_{p} f \\
& =\int_{x} \partial_{t}\left(\alpha \rho_{g} u_{g}\right)+\int_{x, u_{p}, e_{p}} m_{p} u_{p} \partial_{t} f \\
& =-\int \nabla_{x} p+\nabla_{x} \cdot\left(\alpha \rho_{g} u_{g} \otimes u_{g}\right)-\int_{x, u_{p}, e_{p}} m_{p} \Gamma f \\
& \quad-\int_{x, u_{p}, e_{p}}\left(m_{p}\left(u_{p} \cdot \nabla_{x} f\right) u_{p}+m_{p} \partial_{e_{p}}(f \Phi)\right)+\int_{x, u_{p}, e_{p}} m_{p} \Gamma f \\
& =0 .
\end{aligned}
$$

That ends the proof of Proposition 1.

\section{Numerical tests}

3.1. Numerical method. Our method is close to that of [MOT] and was originally developed for a simulation code in the context of the nuclear industry. We use a splitting of the fluid part (2.6)-(2.8) of the model and of its kinetic part (2.10)-(2.12). We refer to [BDM] for more details about the scheme.

3.1.1. Solving the fluid equations. The numerical method for solving (2.6)(2.8) is time-split into two steps, respectively called Lagrange and "re-map" (or transport). In the first step (Lagrange), we solve a system which is quite similar to the Lagrange reformulation of (2.6)-(2.8) (see [GOR]): that means that we solve these equations in a referential which follows the fluid flow. The second step (re-map) gets us back from the Lagrange referential to the fixed one. The reader will find the study of a similar scheme for the compressible Euler equations (without source term) in [DEL].

We here use a finite volume method to discretize this system. This method is space-split itself: every fluid quantity is computed at the center of each volume, except the fluid velocities, which are computed at the borders of each volume.

3.1.2. Solving the kinetic equation. We use a particle method to solve the Vlasov equation (2.12): the PDF $f$ is discretized by a sum of Dirac masses. Consequently, when we need to compute some quantity at point $x$, we are led to use control volumes. The whole physical space is divided into such control volumes that do not depend on the droplets but are a priori defined. For the sake of simplicity, in this code, control volumes are chosen to coincide with the finite volumes used in the solving of the fluid equations.

For instance, if we want to compute the fluid volume fraction at time $t$ and position $x$, we consider the control volume $V$ which contains $x$. Then we replace 
TABLE 3.1. Thermodynamic state of the fluid and the droplets

\begin{tabular}{|c|c|c|c|}
\hline & pressure $\left(\times 10^{6} \mathrm{~Pa}\right)$ & temperature $(\mathrm{K})$ & density $\left(\mathrm{kg} \cdot \mathrm{m}^{-3}\right)$ \\
\hline fluid & 1.00 & 1000 & 0.388 \\
\hline droplet & 1.00 & 100 & 12.1 \\
\hline
\end{tabular}

the Dirac masses (with respect to position) by characteristic functions. Hence, the discretized value of the fluid volume fraction at time $t$ and position $x$ writes

$$
\alpha_{V}(t)=1-\sum_{p} \frac{m_{p}}{\rho_{p}}|V|^{-1} \mathbf{1}_{\left\{x_{p} \in V\right\}},
$$

where one sums over all the droplets inside the computation domain.

3.1.3. Implicit in time feature of the equations. As we already pointed out in Remark 1, the system (2.1)-(2.12) is time-implicit. We noticed that we had to first solve the implicit integral equation (2.13). In theory, (2.13) should be solved by a fixed point method: more precisely, at each time step, this fixed point method should be implemented. However, since we are only interested in weakly compressible droplets, we are led to replace this (very expensive) procedure by the simple approximation

$$
\begin{aligned}
\partial_{t} \rho_{p}\left(t^{n+1}, x, \tilde{e_{p}}\right)= & \int_{u_{p}, e_{p}} \mathcal{F}\left(t^{n}, x, u_{p}, e_{p}, \tilde{e_{p}}\right) \partial_{t} \rho_{p}\left(t^{n}, x, e_{p}\right) f\left(t^{n}, x, u_{p}, e_{p}\right) d u_{p} d e_{p} \\
& +\mathcal{G}\left(t^{n}, x, \tilde{e_{p}}\right) .
\end{aligned}
$$

3.2. Droplets in thermal imbalance with the fluid. In order to test the validity of our code, we consider a situation in which the initial datum does not depend on the variable $x$ (uniform medium): inside each mesh (the volume of which equals to $V=0.025 \mathrm{~m}^{3}$ ), there are $n=5000$ droplets. Both phases are supposed to be ideal gases. The thermodynamic states of the fluid and each droplet are given in table 3.1. Note that the fluid and the droplets have the same pressure because of the isobaric equilibrium assumption.

In this particular case, there exists a semi-explicit solution. As a matter of fact, first, the uniformity with respect to $x$ implies that there is no dependence on $x$ for every studied quantity. Next, since there is initially no motion inside both the spray and the fluid, it is clear that we can take $u_{g}=0$ and that the spray has only one celerity $u_{p}=0$. Then $f$ now only depends on $t$ and $e_{p}$, so that we can choose $f$ as a Dirac mass: $f\left(t, e_{p}\right)=\delta_{e_{p}=e_{p}(t)}$. Finally, we have to note that the equations of state are very simple because both phases are ideal gases. Consequently, all the quantities are algebraic functions of $e_{g}$ and $e_{p}$, which satisfy the following system of ordinary differential equations

$$
\begin{gathered}
e_{g}^{\prime}=-\left(\gamma_{g}-1\right) \frac{\alpha^{\prime}}{\alpha} e_{g}-\frac{1}{M_{g}} \frac{n}{V} 4 \pi r_{p} \lambda \mathrm{Nu}\left(\frac{e_{g}}{c_{v_{g}}}-\frac{e_{p}}{c_{v_{p}}}\right), \\
m_{p} \gamma_{p} e_{p}^{\prime}=4 \pi r_{p} \lambda \mathrm{Nu}\left(\frac{e_{g}}{c_{v_{g}}}-\frac{e_{p}}{c_{v_{p}}}\right)+\left(\gamma_{p}-1\right) m_{p} e_{p}\left(\frac{e_{g}^{\prime}}{e_{g}}-\frac{\alpha^{\prime}}{\alpha}\right),
\end{gathered}
$$




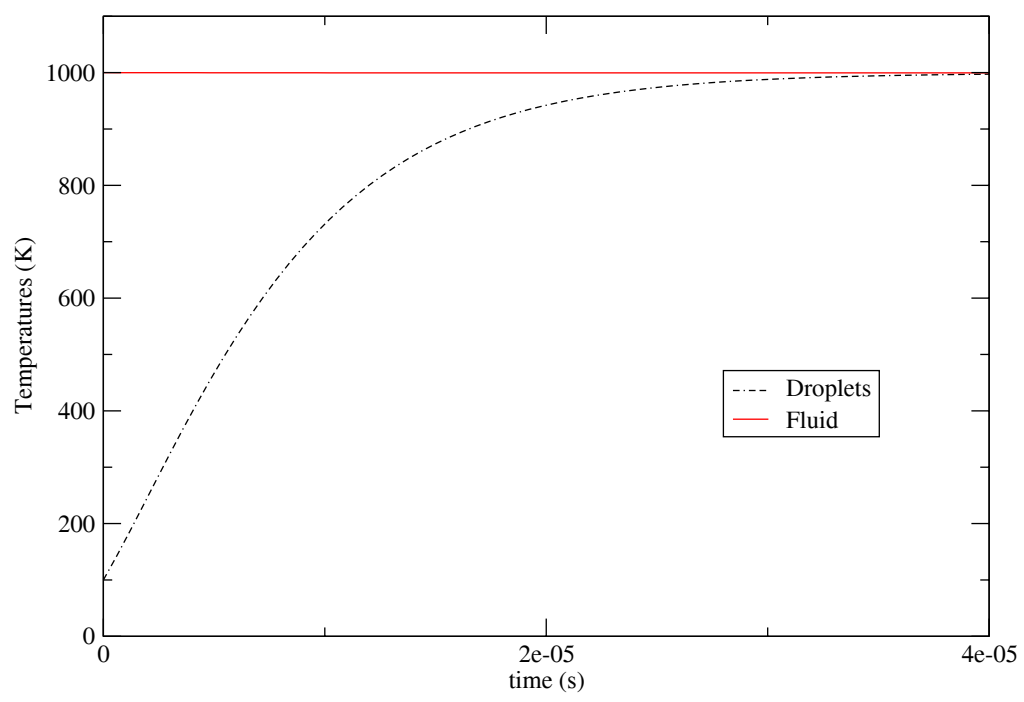

FIG. 3.1. Towards the equilibrium of temperatures.

where $c_{v_{g}}$ (resp. $c_{v_{p}}$ ) is the fluid (resp. droplets) isochoric (that is, when the volume is fixed) specific heat, $\gamma_{g}$ (resp. $\gamma_{p}$ ) the ratio of the fluid (resp. droplets) specific heats, $M_{g}$ is the constant value of $\alpha \rho_{g}$, and where $\alpha$ and $r_{p}$ are given by

$$
\begin{aligned}
\alpha & =\left(1+\frac{n}{V} \frac{\gamma_{p}-1}{\gamma_{g}-1} \frac{m_{p}}{M_{g}} \frac{e_{p}}{e_{g}}\right)^{-1}, \\
r_{p} & =\left(\frac{3}{4 \pi} \frac{\gamma_{p}-1}{\gamma_{g}-1} \frac{\alpha m_{p}}{M_{g}} \frac{e_{p}}{e_{g}}\right)^{1 / 3} .
\end{aligned}
$$

Two physical phenomena happen almost simultaneously:

- the temperatures of both fluid and droplets are well-balanced (see fig. 3.1);

- to balance the increasing of the temperature, each droplet density decreases to reach an equilibrium value (see fig. 3.2).

Fig. 3.2 also shows that the numerical results obtained thanks to our code fit very well to the semi-explicit solutions (computed by Maple).

This test was very accurate to get both mathematical and programming validations of the model. Still the thermodynamics lead to very important and unrealistic variations of the densities of each droplet. The following computation has been performed in a more realistic thermodynamic setting.

3.3. Hollow-cone spray. This second numerical test has been set in [DUA] and allows us to compare the numerical results for both compressible and incompressible droplets. The droplets are injected with the same velocity $100 \mathrm{~m} \cdot \mathrm{s}^{-1}$ in a 

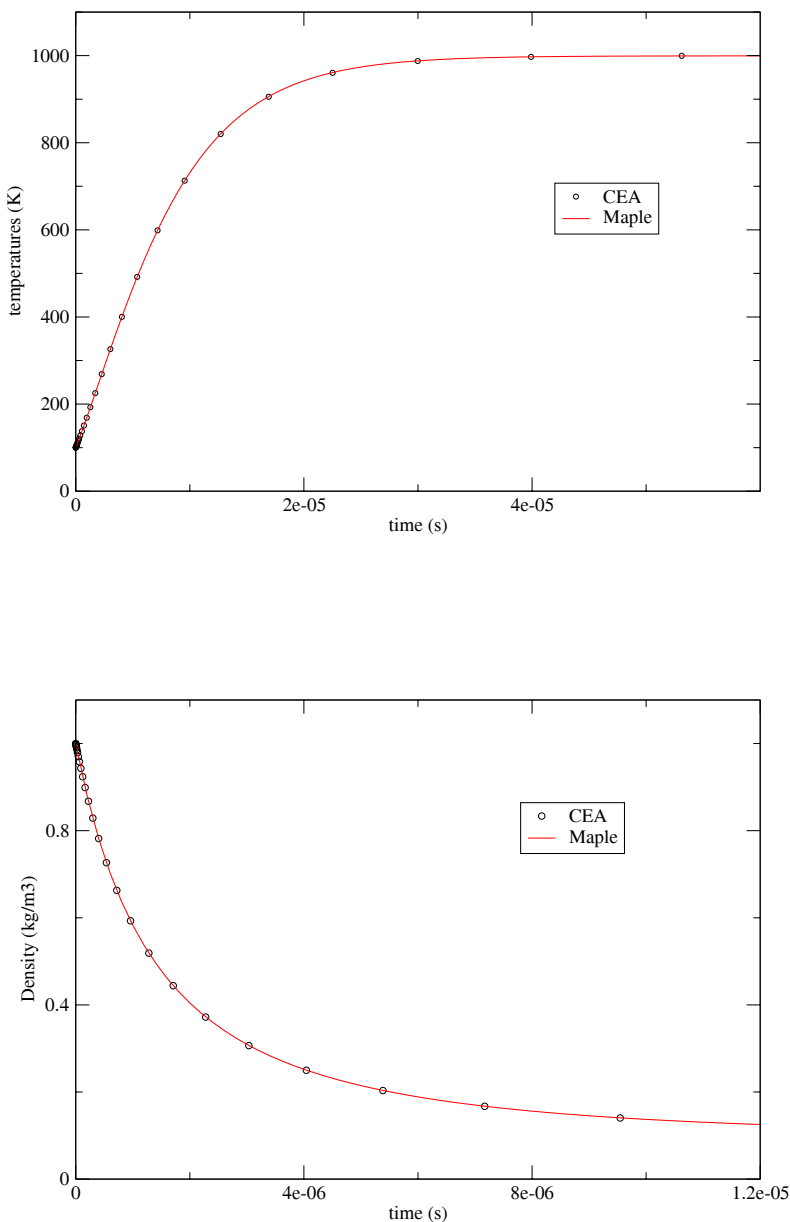

FIG. 3.2. Temperature and density of a droplet.

fluid following the border of a hollow-cone (with angle $45^{\circ}$ ). The fluid is initially not moving and has a higher temperature than the droplets $(500 \mathrm{~K}$ for the fluid, $300 \mathrm{~K}$ for the spray). Both phases satisfy realistic equations of state (stiffened gas for the fluid, standard tin for the spray).

Fig. 3.3 describes the occurring phenomenon: the droplets make the fluid move and cool down. We here propose the evolution of a sample droplet: densities (see fig. 3.4) and temperatures (see fig. 3.5). In fig. 3.4, the density of the incompressible droplet remains constant, whereas the one of the compressible droplet decreases to balance the increasing of the droplet temperature. The analysis of fig. 3.5 is a bit more intricate: the curve for the compressible droplet is behind the one for the incompressible droplet. As a matter of fact, since there is first the mechanical reaction of the compressible droplet (increasing radius), its temperature does not increase as 


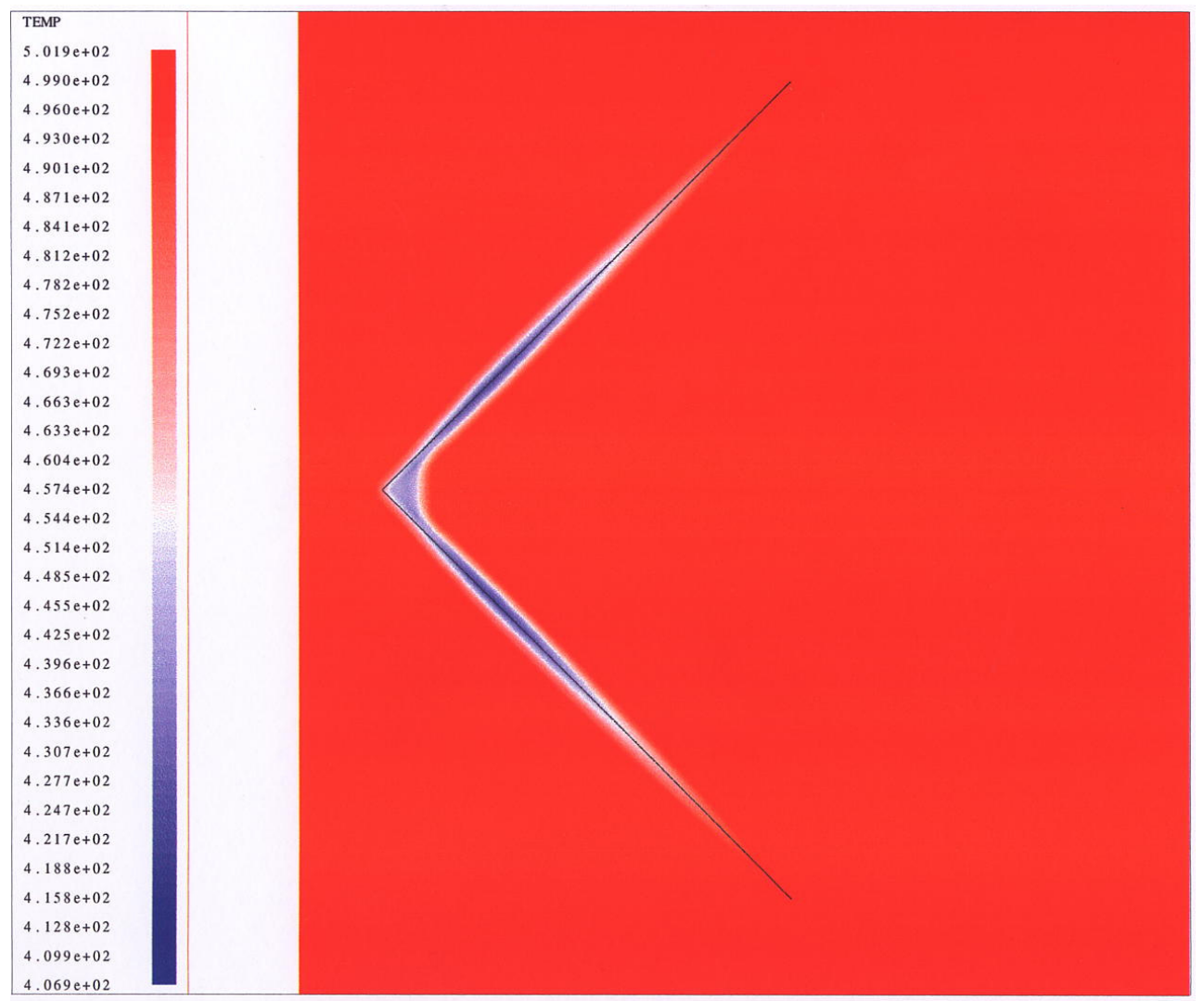

FIG. 3.3. Evolution of the fluid temperature due to the droplets.

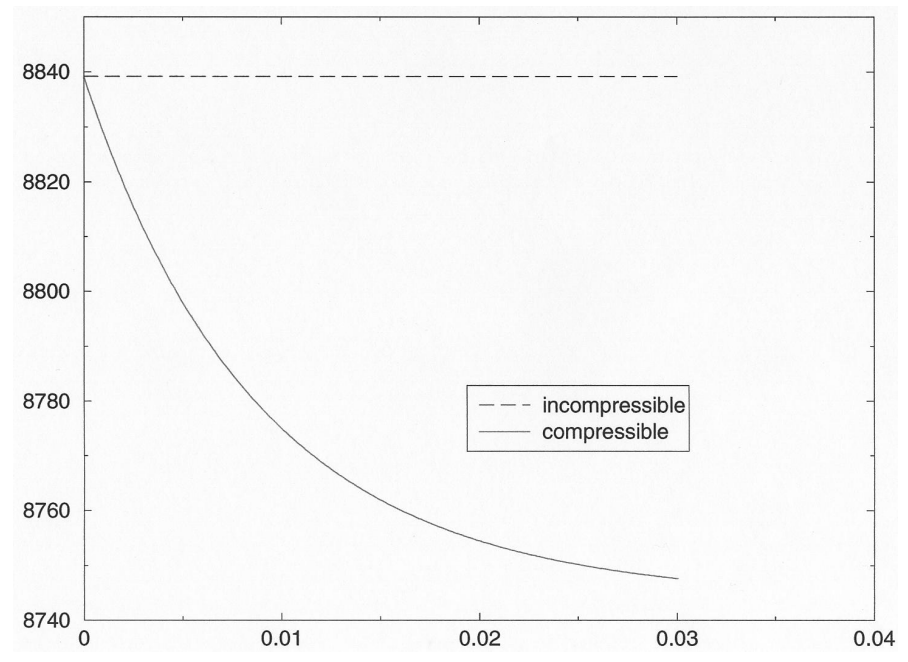

FIG. 3.4. Density of the sample droplet. 


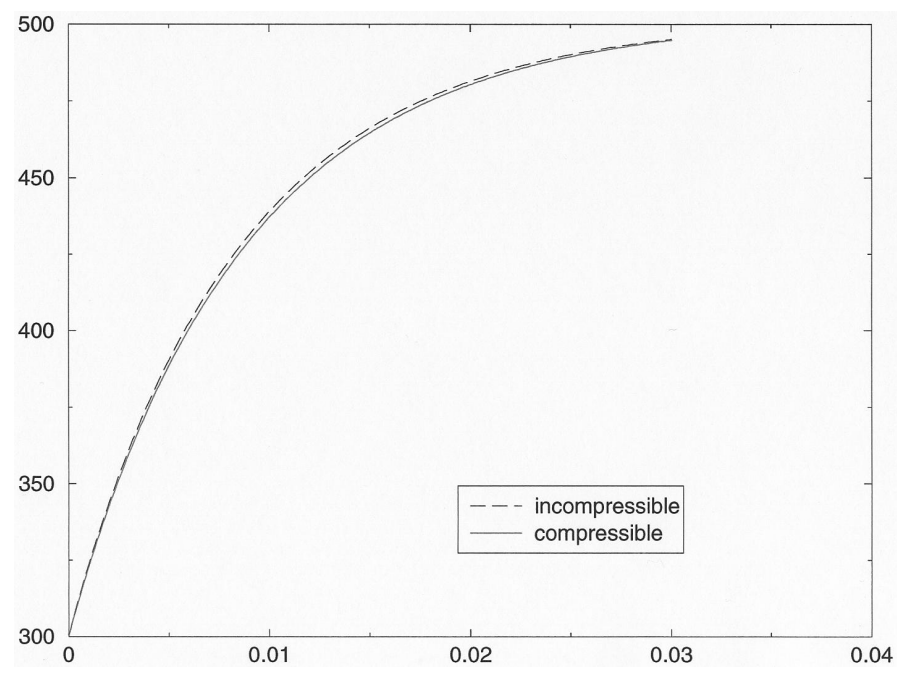

FIG. 3.5. Temperature of the sample droplet.

fast as the temperature of the incompressible droplet. And we already pointed out in subsection 2.1 that mechanical phenomena occur faster than thermodynamic ones. But eventually, we note that for both figures, there is approximately a $1 \%$ difference between the incompressible and compressible curves.

In fact, we noticed in other numerical tests that, in the context of the nuclear industry, the density of a given droplet typically varies by $1 \%$ during the evolution of the spray, so that we can state that the effects of the compressibility of the droplets seem to be small for realistic thermodynamics. Therefore, the intricate model studied here should be used only when very precise results are needed. On the other hand, the numerical comparison is not much more expensive when the compressibility is taken into account, because of this small variation of the density of a droplet. As a matter of fact, one solves the implicit equations only approximately, and with a fine accuracy.

Appendix: value of the physical coefficients. To complete our modeling, we give the value of every coefficient which appears in (2.8)-(2.11). The physical justifications of our choices can be found in [RAN], for instance.

First of all, let us give some precisions about the drag coefficient $C_{d}$, the added mass coefficient $C_{a}$ and the Nusselt number Nu.

We empirically fix the coefficient $C_{a}=0.5$.

To write $C_{d}$ and $\mathrm{Nu}$, let us begin to recall the definition of some standard coefficients, such as the Reynolds, Mach and Prandtl numbers

$$
\operatorname{Re}=\frac{2 r_{p} \rho_{g}\left|u_{p}-u_{g}\right|}{\eta}, \quad \mathrm{Ma}=\frac{\left|u_{p}-u_{g}\right|}{c}, \quad \operatorname{Pr}=\frac{\eta C}{\lambda},
$$

where the fluid viscosity $\eta$ is a given constant, $c$ is the local sound velocity in the fluid and the fluid specific heat $C$ and thermal conductivity $\lambda$ are given.

We then take for $C_{d}$ the formula

$$
C_{d}=\tilde{C}_{d}(\mathrm{Re}) \tilde{\theta}(\mathrm{Ma}),
$$


with

$$
\begin{aligned}
& \tilde{C}_{d}=\frac{24}{\operatorname{Re}}\left(\alpha^{-2.65}+\frac{1}{6} \operatorname{Re}^{2 / 3} \alpha^{-1.78}\right) \text { if } \operatorname{Re}<\operatorname{Re}_{c}:=1000 \\
& \tilde{C}_{d}=\frac{24}{\operatorname{Re}_{c}}\left(\alpha^{-2.65}+\frac{1}{6} \operatorname{Re}^{2 / 3} \alpha^{-1.78}\right) \text { if } \operatorname{Re}>\operatorname{Re}_{c}
\end{aligned}
$$

and

$$
\begin{aligned}
& \tilde{\theta}=1 \quad \text { if } \mathrm{Ma}<0.5 \text {, } \\
& =2.22 \quad \text { if } \mathrm{Ma}>1 \text {, } \\
& =2.44 \mathrm{Ma}-0.22 \text { if } 0.5<\mathrm{Ma}<1 \text {. }
\end{aligned}
$$

We also take for the Nusselt number the formula

$$
\mathrm{Nu}=\alpha^{-7 / 4}+0.3 \operatorname{Re}^{1 / 2} \operatorname{Pr}^{1 / 3} \alpha^{-1 / 2} .
$$

Finally, the term $D_{p}$ in the expression of the drag force is fixed by the expression

$$
D_{p}=\frac{1}{2} \pi r_{p}^{2} \rho_{g} C_{d}\left|u_{p}-u_{g}\right|
$$

\section{REFERENCES}

[KIV] A.A. Amsden, P.J. O'Rourke, and T.D. Butler, A computer program for chemically reactive flows with sprays, Report \# LA-11560-MS, Los Alamos National Laboratory, 1989.

[KIW] A.A. Amsden, Kiva-3V, release 2, improvements to Kiva-3V, Report \# LA-UR-99-915, Los Alamos National Laboratory, 1999.

[BAA] C. Baranger, Modelling of oscillations, breakup and collisions for droplets: the establishment of kernels for the T.A.B. model, Preprint CMLA, 2003.

[BDM] L. Boudin, L. Desvillettes, and R. Motte, A particle-gas model for compressible droplets, in the proceedings of the conference "Trends in numerical and physical modeling for industrial multiphase flows", Cargèse, France, 2000.

[CEC] C. Cercignani, The Boltzmann equation and its applications, Applied Mathematics, vol. 67, Springer-Verlag, 1988.

[CLG] R. Clift, J.R. Graces, and M.E. Weber, Bubbles, Drops and Particles, Academic Press, 1978.

[DEL] B. Després and F. Lagoutière, Contact discontinuity capturing schemes for linear advection and compressible gas dynamics, J. Sci. Comput., 16(4):479-524, 2002.

[DOA] K. Domelevo, Mathematical and numerical analysis of a kinetic modeling of a spray, $\mathrm{PhD}$ thesis, École Polytechnique, 1996.

[DRA] D.A. Drew, Mathematical modeling of two-phase flow, Rev. Fluid Mech., 15:261-291, 1983.

[DUA] J.K. Dukowicz, A particle-fluid numerical model for liquid sprays, J. Comp. Phys., 35:229$253,1980$.

[GOR] E. Godlewski and P.A. Raviart, Numerical Approximation of Hyperbolic Systems of Conservation Laws, Springer-Verlag, 1995.

[LAN] L. Landau and E. Lifchitz, Physique Théorique tome 5: Physique Statistique, Éditions Mir, 1967.

[MOT] R. Motte, A numerical method for solving particle-fluid equations, in the proceedings of the conference "Trends in numerical and physical modeling for industrial multiphase flows", Cargèse, France, 2000.

[ORO] P.J. O'Rourke, Collective drop effects on vaporizing liquid sprays, PhD thesis, Los Alamos National Laboratory, 1981.

[RAN] W.E. Ranz and W.R. Marshall, Evaporization from drops, part I-II, Chem. Eng. Prog., 48(3):141-180, 1952

[RAV] P.A. Raviart and L. Sainsaulieu, A nonconservative hyperbolic system modeling spray dynamics part 1: solution of the Riemann problem, Math. Models and Meth., in Applied Science, 5(3):297-333, 1995.

[SAA] L. Sainsaulieu, Equilibrium velocity distribution functions for a kinetic model of two-phase fluid flows, Math. Models and Meth., in Applied Science, 5(2):191-211, 1995.

[MAS] M. Massot and P. Villedieu, Modélisation multi-fluide eulérienne pour la simulation de brouillards denses polydispersés, C. R. Acad. Sci., Paris, Sér. I, Math., 332(9):869-874, 2001.

[WIA] F.A. Williams, Combustion Theory, second edition, Benjamin Cummings, 1985. 